\title{
Salmonelles et SAuCisses À la Réunion
}

\author{
Salmonella and sausages in ReUnion IsLAND
}

Salmonella y SALChichas eN LA ISLA DE LA ReUnión

\author{
A. Trimoulinard ${ }^{1}$ C. Tessier ${ }^{1,2}$ L. Atiana ${ }^{1}$ E. Cardinale ${ }^{1,3 *}$
}

\section{Mots-clés : Viande porcine - Viande de volaille - Salmonella - Facteur de risque - Contamination - Réunion.}

Keywords: Pork - Poultry meat - Salmonella - Risk factor Contamination - Reunion.

Palabras clave: Carne de cerdo - Carne de ave - Salmonella Factor de riesgo - Contaminación - Reunión.

es Réunionnais consomment beaucoup de viande de volaille Let de porc, et les saucisses 100 p. 100 volaille et 100 p. 100 porc figurent parmi les particularités notables de la cuisine locale. Une enquête chez les professionnels de la charcuterie et des analyses bactériologiques ont permis de déterminer les facteurs qui pouvaient favoriser la contamination de ces produits à l'étape de la vente.

Au total 203 échantillons de saucisses de porc et de volaille ont été prélevés dans 67 points de vente (supermarchés ou hypermarchés, épiceries, et boucheries-charcuteries), tirés aléatoirement sur l'ensemble de l'île de la Réunion. A partir d'analyses bactériologiques, les prévalences de Salmonella spp. et de Campylobacter spp. ont été déterminées ainsi que les sérotypes majeurs de Salmonella; la population de Salmonella dans les saucisses de volaille et de porc a aussi été quantifiée. Les analyses bactériologiques ont été réalisées selon les normes européennes. Les pratiques à risque conduisant à une contamination des produits consommés ont été identifiées à partir d'une enquête d'observation relative aux pratiques de vente et d'un modèle linéaire généralisé sous une loi binomiale.

Des prévalences faibles de Salmonella spp. ont été observées pour les lots de saucisses et pour les points de vente. Salmonella spp. n'a été détectée que dans 11,8 p. 100 de ces échantillons (95 p. 100 intervalle de confiance $=7,8-17,3$ ). Cette prévalence était différente en fonction du type de point de vente et des caractéristiques de la saucisse (tableau I) : les saucisses de porc, les saucisses fumées et les saucisses « fraiches » (reconstituées à partir de viande congelée-décongelée) ont été plus contaminées (test $\mathrm{Z}, \mathrm{p}<0,001$ ).

1. Cirad, UMR Cmaee, Centre de recherche et de veille sur les maladies émergentes dans l'océan Indien, 2 rue Maxime Rivière, BP 80005, 97491 Sainte-Clotilde Cedex, Réunion, France.

2. Coopérative des porcs de la Réunion, 5 av. Charles Isautier, ZI n 3, 97410 SaintPierre, Réunion, France.

3. Centre de recherche et de veille sur les maladies émergentes dans l'océan Indien, Cyroi, Réunion, France.

* Auteur pour la correspondance

E-mail : eric.cardinale@cirad.fr

\section{Tableau I}

Contamination par Salmonella spp. observée selon les caractéristiques des saucisses dans divers points de vente de la Réunion

\begin{tabular}{|c|c|c|}
\hline \multirow[t]{2}{*}{ Variable } & \multicolumn{2}{|c|}{ Prévalence de Salmonella (S+) } \\
\hline & $\%$ (nb.) & IC $95 \%$ \\
\hline Saucisse & $11,82(24 / 203)^{a}$ & $7,80-17,32$ \\
\hline Saucisse de porc & $16,53(21 / 127)$ & $10,07-23,00$ \\
\hline Saucisse de volaille & $3,95(3 / 76)$ & $0,00-8,33$ \\
\hline Saucisse fumée & $11,11(10 / 90)$ & $4,62-17,60$ \\
\hline Saucisse non fumée & $12,39(14 / 113)$ & $6,31-18,46$ \\
\hline Saucisse fraîche & $17,14(24 / 140)$ & $10,90-23,39$ \\
\hline Saucisse conditionnée & $0,00(0 / 55)$ & $0,00-8,13$ \\
\hline $\begin{array}{l}\text { Saucisse fabriquée localement } \\
\text { (viande importée) }\end{array}$ & $12,31(24 / 195)$ & $7,70-16,92$ \\
\hline
\end{tabular}

IC : intervalle de confiance

${ }^{a}$ Différence significative entre les points de vente avec $p<0,01$, test $Z$

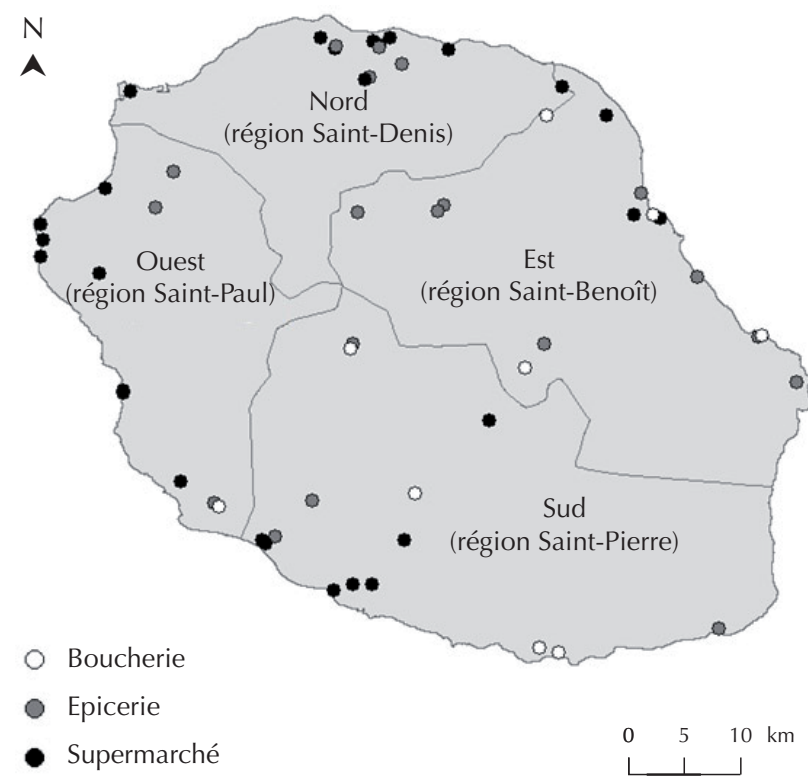

Figure 1 : identification des points de vente considérés dans l'étude sur la contamination des saucisses de volailles et de porcs par les salmonelles à la Réunion (2012). 
Les sérovars détectés selon les lots de saucisses ont été S. Typhimurium (4,92 p. 100), S. London (2,46 p. 100), S. Derby (1,98 p. 100), S. Newport (0,99 p. 100), S. Blockley (0,49 p. 100) et $S$. Weltevreden $(0,49$ p. 100). La moyenne de population de Salmonella spp. par échantillon a été de 72,9 bactéries/g avec un minimum de 6,00 bactéries/g et un maximum de 380 bactéries/g, soit des niveaux largement en deçà de la dose infectieuse. La vente de saucisses dans un sac en plastique (odds ratio $[\mathrm{OR}]=26,63)$, dans un emballage en papier $(\mathrm{OR}=9,00)$ et l'absence de lutte contre les rongeurs $(O R=5,42)$ ont été corrélées positivement au risque de contamination par Salmonella spp. Une surface de vente importante $\left(>250 \mathrm{~m}^{2}\right)(\mathrm{OR}=0,99)$ a diminué ce risque. Un pourcentage des lots de saucisses a été contaminé par Campylobacter spp. Aucun facteur de risque ou de protection vis-à-vis de Campylobacter n'a été déterminé car la prévalence a été trop faible pour l'associer à des pratiques de fabrication des saucisses. Le risque pour le consommateur reste limité puisque les saucisses sont bien cuites dans les carrys et autres plats traditionnels.

Les gérants des points de vente peuvent donc accentuer leurs efforts, d'une part, en utilisant des détergents et des désinfectants pour le nettoyage des vitrines et, d'autre part, en lavant régulièrement les vêtements de travail du personnel. II importe enfin d'assurer des formations de base en hygiène pour le personnel. Les gérants doivent également insister sur leur méthode de conditionnement. Même si des salmonelles et des campylobacters sont régulièrement identifiés en élevage $(1,2)$, les prévalences et les doses observées à la mise en marché ne risquent pas de provoquer de gastro-entérites chez le consommateur.

\section{BIBLIOGRAPHIE}

1. CARDinAle E., MAEDER S., PORPHYRE V., DEBIN M., 2010. Salmonella in fattening pigs in Reunion Island: Herd prevalence and risk factors for infection. Prev. Vet. Med., 96: 281-285.

2. HENRY I., GRANIER S., COURTILLON C., LALANDE F., CHEMALY M., SALVAT G., CARDINALE E., 2012. Salmonella enterica subsp. enterica isolated from chicken carcasses and environment at slaughter in Reunion Island: prevalence, genetic characterization and antibiotic susceptibility. Trop. Anim. Health Prod., 45: 317-326.

Accepted 30 April 2015; Online publication June 2015 